\title{
Development of a new algorithm for segmentation of flotation froth images
}

\begin{abstract}
It is well known that froth visual features reflect the operating conditions of the flotation process, so that being able to accurately obtain the froth properties is the most significant criteria to optimize and control this process. Froth segmentation is a useful procedure that can determine the bubble size distribution. Several algorithms have been proposed in this field, but marker-based watershed transform shows the best performance. In spite of this fact, the algorithm suffers from oversegmentation in cases when the flotation froth includes large bubbles along with small ones. In the paper, the marker-based watershed method is modified to prevent oversegmentation of large bubbles. The developed algorithm is validated using some froth images captured at different operating conditions, so the results indicate that the method can segment the mixture of big and small bubbles effectively.
\end{abstract}

Keyword: Algorithm; Flotation; Froth images; Bubble size 